\title{
HEAVY-ION IRRADIATION INDUCED DIAMOND FORMATION IN CARBONACEOUS MATERIALS*
}

\author{
T. L. Daulton, L. E. Rehn, and M. A. Kirk \\ Materials Science Division \\ 9700 S. Cass Avenue \\ Argonne National Laboratory
}

Argonne, IL 60439

R. S. Lewis

Enrico Fermi Institute

University of Chicago

Chicago, IL 60637-1433

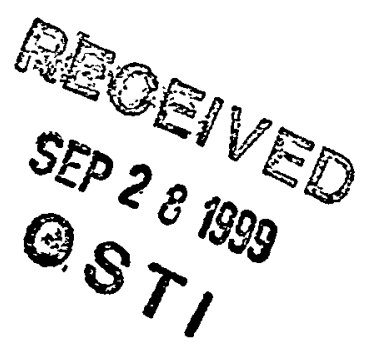

December 1998

The submitted manuscript has been created
by the University of Chicago as Operator on
Argonne National Laboratory (Argonne'
under Contract No. W-31-109-ENG 38 with
the U.S. Department of Energy. The U.S.
Government retains for itself, and others
acting on its behalf, a paid-up, non
exclusive, irrevocable worldwide license in
said article to reproduce, prepare derivative
works, distribute copies to the public, and
perform publicly and display publicly, by or
on behalf of the Govemment.

To be submitted to the Materials Research Society, November 30-December 4, 1998, Boston, MA.

\footnotetext{
*Work supported by the U.S. Department of Energy, Basic Energy Sciences-Materials Sciences, under contract \#W-31-109-ENG-38 and NASA under grant NAG5-4297.
} 


\section{DISCLAIMER}

This report was prepared as an account of work sponsored by an agency of the United States Government. Neither the United States Government nor any agency thereof, nor any of their employees, make any warranty, express or implied, or assumes any legal liability or responsibility for the accuracy, completeness, or usefulness of any information, apparatus, product, or process disclosed, or represents that its use would not infringe privately owned rights. Reference herein to any specific commercial product, process, or service by trade name, trademark, manufacturer, or otherwise does not necessarily constitute or imply its endorsement, recommendation, or favoring by the United States Government or any agency thereof. The views and opinions of authors expressed herein do not necessarily state or reflect those of the United States Government or any agency thereof. 


\section{DISCLAIMER}

Portions of this document may be illegible in electronic image products. Images are produced from the best available original document. 


\title{
HEAVY-ION IRRADIATION INDUCED DIAMOND FORMATION in Carbonaceous Materials
}

\author{
T. L. Daulton ${ }^{1}$, R. S. Lewis ${ }^{2}$, L. E. Rehn ${ }^{1}$, and M. A. Kirk ${ }^{1}$ \\ ${ }^{1}$ Materials Science Division, Argonne National Laboratory, Argonne IL, 60439. \\ ${ }^{2}$ Enrico Fermi Institute, University of Chicago, Chicago IL, 60637.
}

\begin{abstract}
The basic mechanisms of metastable phase formation produced under highly non-equilibrium thermodynamic conditions within high-energy particle tracks are investigated. In particular, the possible formation of diamond by heavy-ion irradiation of graphite at ambient temperature is examined. This work was motivated, in part, by earlier studies which discovered nanometer-grain polycrystalline diamond aggregates of submicron-size in uranium-rich carbonaceous mineral assemblages of Precambrian age. It was proposed that the radioactive decay of uranium formed diamond in the fission particle tracks produced in the carbonaceous minerals. To test the hypothesis that nanodiamonds can form by ion irradiation, fine-grain polycrystalline graphite sheets were irradiated with $400 \mathrm{MeV} \mathrm{Kr}$ ions. The ion irradiated graphite (and unirradiated graphite control) were then subjected to acid dissolution treatments to remove the graphite and isolate any diamonds that were produced. The acid residues were then characterized by analytical and high-resolution transmission electron microscopy. The acid residues of the ion-irradiated graphite were found to contain ppm concentrations of nanodiamonds, suggesting that ion irradiation of bulk graphite at ambient temperature can produce diamond.
\end{abstract}

\section{INTRODUCTION}

The direct transformation of graphitic carbon into diamond is of considerable technological interest. The high stability of the basal graphite planes requires thermodynamic conditions deep within the diamond phase region or the presence of catalysts for solid-state transformation of graphite into diamond. For example, transformation of graphite to diamond has been achieved using high static pressure [1] and dynamic shock [2]. It has been proposed that point-defects produced by ion-irradiation could lower the free energy barrier for transformation [3]. Such arguments have motivated numerous attempts to transform graphite into diamond using irradiation. However, until recently, all attempts have been unsuccessful. The only two successful attempts were elevated temperature $\mathrm{MeV}$ electron [4] and ion [5] irradiations of carbon onions (concentric graphitic shelled structures of nanometer diameter) have been reported to convert the cores of the onions into diamond. It was proposed that the combination of particle irradiation and annealing at elevated temperature causes a significant contraction in the spacings of the concentric shells. This, in turn, generates pressures in the core above the graphite/diamond equilibrium phase line, permitting solid-state transformation of graphite into diamond $[4,5]$.

The free-energy difference between diamond and graphite is very small, and under appropriate conditions, both allotropes will form. There is evidence suggesting that graphite can directly recrystallize as diamond in ion-particle tracks without the need of annealing at elevated temperature. The discovery of $30 \mathrm{ppm}$ concentrations of nanodiamond aggregates in crustal Urich coal-like carbonaceous assemblages of Precambrian age suggests direct recrystallization of diamond in the cores of $U$ fission tracks produced in the carbonaceous minerals [6]. The nanodiamonds in these assemblages were revealed only upon their isolation from the bulk by acid 
dissolution treatments. If they were produced by ion irradiation, this suggests a low formation efficiency which could contribute to the failure to observe diamond in well-studied ion-implanted . carbons or in unsuccessful irradiation experiments which attempted diamond formation.

In this work, we test if nanodiamonds can form in bulk graphite irradiated by heavy highenergy ions at ambient temperature. For our experiments, we selected ion-irradiation conditions similar to those experienced in the nanodiamond containing U-rich carbonaceous assemblages. There are three main decay products of ${ }^{238} \mathrm{U} ; \alpha$-particles $(\approx 4 \mathrm{MeV}), \alpha$-recoil nuclei $(\approx 100 \mathrm{keV})$ and $\mathrm{Kr}$ and $\mathrm{Xe}$ fission fragments $(\approx 100 \mathrm{MeV})$. Both the $\alpha$-recoil nuclei and fission fragments produce substantial lattice displacement damage. The rate of ${ }^{238} U \alpha$-decay is over six orders of magnitude greater than that of spontaneous fission into two heavy fragments. However, the range of $\alpha$-recoil nuclei is very short $(\approx 10 \mathrm{~nm})$ and likely does exceed that necessary to escape U-oxide grains and interact with carbonaceous material. Therefore, in the U-rich carbonaceous, assemblages it is mostly likely the fission fragments which could produce the diamond. In our experiments, fine-grain polycrystalline bulk graphite is irradiated by $400 \mathrm{MeV} \mathrm{Kr}$ ions at ambient temperature. This work is unique in that the irradiated graphite is subsequently processed by acid dissolution treatments designed to recover ppm concentrations of nanodiamonds. These acid residues are characterized using transmission electron microscopy. In this paper we discuss the preliminary results of our ion irradiations and subsequent acid dissolutions of bulk graphite.

\section{EXPERIMENT}

Fine grain polycrystalline graphite sheets of $125 \mu \mathrm{m}$ thickness and $99.95 \%$ carbon purity were cut into 1.14 by $1.59 \mathrm{~cm}$ pieces. Ion irradiations were performed at the Argonne Tandem Linear Accelerator System (ATLAS) at Argonne National Laboratory (ANL) using the large scattering facility. The graphite pieces were mounted on $\mathrm{Cu}$ heat sinks cooled to $20^{\circ} \mathrm{C}$. The specimens were irradiated individually by $400 \mathrm{MeV} \mathrm{Kr}$ to a fluence of $6 \times 10^{12}$ ion-cm-2 on both sides. A gold scattering foil was placed in the beam path to spread the ion beam so that the entire exposed surface of the specimen $\left(1.5 \mathrm{~cm}^{2}\right)$ was irradiated. This resulted in a dispersion and reduction in the energies of the $\mathrm{Kr}$ ions to $\approx 350 \pm 50 \mathrm{MeV}$. The ranges of these ions were estimated to be $35 \pm 5 \mu \mathrm{m}$ from Monte Carlo based computer algorithms, TRIM, using the binary collision approximation [7]. Therefore, about $40 \%$ of the bulk of each specimen received ion irradiation.

Both unirradiated $(0.339 \mathrm{~g})$ and ion-irradiated $(0.317 \mathrm{~g})$ graphite were separately subjected to the standard acid dissolution procedure developed to recover $300 \mathrm{ppm}$ concentrations of nanodiamonds from primitive carbonaceous meteorites [8]. The basic procedure involves the oxidation of graphitic carbons by gradual heating $\left(100^{\circ} \mathrm{C} / \mathrm{hr}\right.$ up to $\left.200^{\circ} \mathrm{C}\right)$ in a solution of (7:2:1) $\mathrm{HClO}_{4}, \mathrm{H}_{2} \mathrm{SO}_{4}$, and $\mathrm{HNO}_{3}$ also containing $0.003 \mathrm{M} \mathrm{Cr}^{+6}$ (which acted as a catalyst). This procedure was applied twice. Following the first oxidation of the ion-irradiated graphite, there was $<1 \mu \mathrm{g}$ of total material remaining. A valuable tool in the separation process is that colloidal properties of nanodiamonds can be controlled by the $\mathrm{pH}$ of their solution and this is usually attributed to the presence of acidic carboxyl groups $(-\mathrm{COOH})$ on their surface [9]. Any nanodiamonds present following both oxidations were placed in colloidal suspension using $0.1 \mathrm{~N}$ $\mathrm{NH}_{4} \mathrm{OH}$, and centrifugation was used to separate them from whatever coarse particles survived (or were contaminants during the processing). After the coarse particles were removed, nanodiamonds were then recovered from the suspension by adding $\mathrm{HCl}$ until the solution $\mathrm{pH}<1$, waiting for the diamonds to aggregate together, and centrifugation. Following this purification step, both samples had $\ll 1 \mu \mathrm{g}$ remaining. Any diamonds present in these residues were 


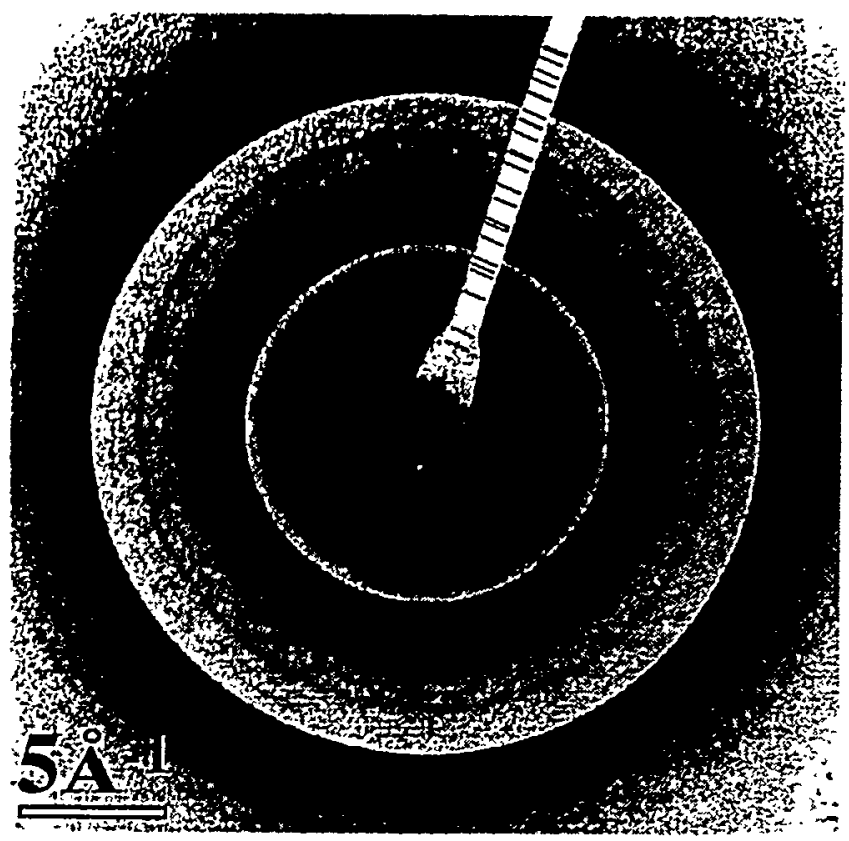

Figure 1: Electron diffraction pattern from a collection of nanocrystals in the acid residues of the ionirradiated graphite. To illustrate all the reflections, three intensity scale changes were applied to the image.

ultrasonically suspended in $12 \mu \mathrm{L}$ of $4: 1$ mixture of isopropanol and $\mathrm{H}_{2} \mathrm{O}$. They were placed into colloidal suspension by adding $\mathrm{HCl}$ until the solution $\mathrm{pH} \sim 1$ or 2 . 1-2 $\mu \mathrm{l}$ aliquots of this solution were deposited on $3 \mathrm{~mm} \mathrm{Cu}$ TEM grids coated with either holey amorphous-C $(\mathrm{a}-\mathrm{C})$ film used for imaging or amorphous-SiO (a-SiO) film used for analytical measurements.

The samples were then characterized by transmission electron microscopes (TEMs) in the Electron Microscopy Center at ANL. Lattice imaging was performed using a JEM JEOL-4000EX high-resolution TEM. Analytical elemental analysis of the residues was performed using a Philips CM30 TEM equipped with a Gatan Model 666 Parallel Electron Energy Loss Spectrometer (PEELS) and an EDAX EDAM-IIs Energy Dispersive $\mathrm{X}$-ray Spectrometer (EDXS).

\section{RESULTS}

The acid residues of the ion-irradiated graphite were found to contain nanocrystals which were dispersed nonuniformly on the support film. Some regions on the support film contained dispersions of nanocrystals with a sufficient number density to record electron diffraction patterns (Figure 1). Figure 2 illustrates a plot of the radial average of the intensity of the electron

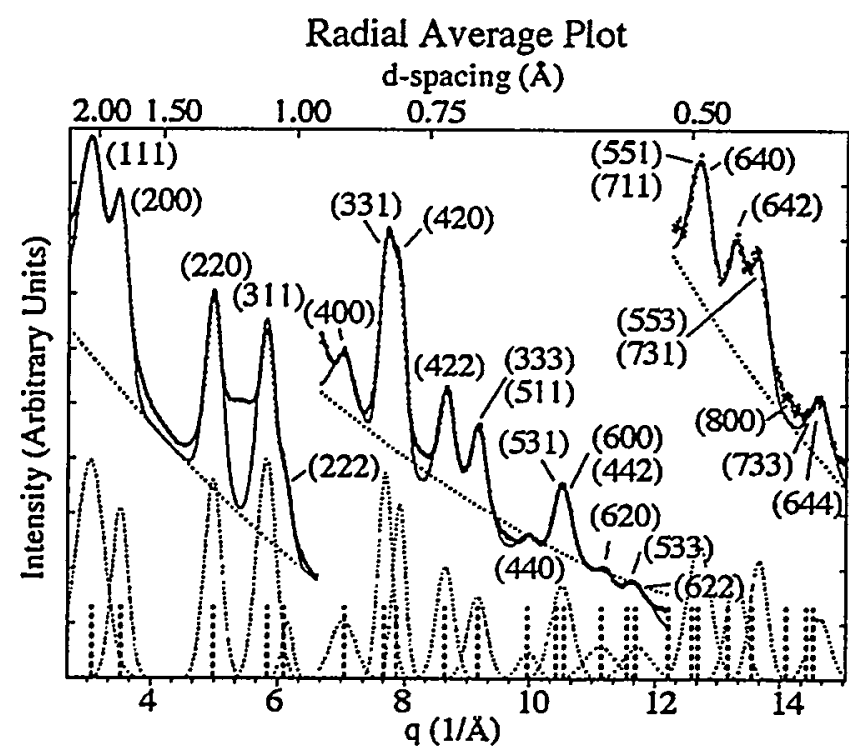

Figure 2: Radial average of intensity in Figure 1. The deconvoluted peaks are shown at the bottom (dashed). The fitted background (dashed curve) and fitted peaks (solid curve) are superimposed on the data. The vertical dashed lines along the abscissa represent predicted diamond reflections. diffraction pattern shown in Figure 1. A comparison of the measured peak positions and the predicted d-spacings of diamond is shown in Table I. The measured diffraction maxima are found to index well to cubic diamond. In addition to the allowed reflections, the kinematically forbidden (200), (222), and (420) cubic diamond reflections are also present with appreciable intensities. The kinematically forbidden reflections with mixed indices (110), (221), and (321) are notably absent. The presence of kinematically forbidden diamond reflections have been previously explained in terms of dynamical diffraction effects and lattice distortions arising from impurity atoms [10-12]. The forbidden reflections observed here result from double diffraction arising from twinning (Figure 3). Double diffraction can readily explain both the presence of forbidden reflections with all even 
indices and the absence of those with mixed indices.

High-resolution lattice images of nanocrystals in the irradiated graphite acid residue show that many exhibit cross lattice fringes consistent with the [011] zone of diamond (Figure 3). The measured lattice plane spacings and angles match those of diamond to within measurement error. The nanocrystal shown in Figure 3 has two (111) stacking-fault planes forming a twin lamallae. The interface at a twin boundary represents one of the lowest-energy lattice defects. As a result, nanocrystals can form twin structures relatively easily to accommodate growth constraints [13]. Many of the nanocrystals in the irradiated graphite acid residues were heavily twinned along $\langle 111\rangle$ planes, accounting for the double diffraction observed.

... The phase contrast produced in high-resolution TEM lattice images is highly dependent on several instrumental parameters and on factors such as specimen thickness and orientation. In general, the relationship between the image contrast and the atomic structure is not straightforward. For example, under certain conditions two different atomic structures can produce similar lattice images. However, changes in the defocus will affect the lattice images from dissimilar structures differently. Therefore to identify a crystal structure, it is necessary to image under a range of defocus conditions and compare them to image simulations.

The behavior of the image contrast of the nanocrystals as a function of defocus was examined and compared to that predicted by multi-slice simulations of diamond. For the [011] zone of diamond, either the unresolved columns of (400)-spaced atom-pairs or the open channels in the structure are imaged as bright dots, depending on the defocus. Since the geometric arrangement of these are similar, a contrast reversal is observed in changing defocus (Figure 4). A

\begin{tabular}{|c|c|c|c|}
\hline \multicolumn{4}{|c|}{$\begin{array}{c}\text { Table I } \\
\text { Electron Diffraction Spacings } \\
\text { Acid Residue of Ion-Irradiated Graphite }\end{array}$} \\
\hline$(\mathrm{h} \mathrm{kl})$ & $\begin{array}{l}\text { Data } \\
(\AA)\end{array}$ & $\begin{array}{l}\text { Diamond } \\
(\AA)\end{array}$ & $\begin{array}{c}\Delta \\
(\AA)\end{array}$ \\
\hline 111 & 2.067 & 2.059 & -0.008 \\
\hline $200 \dagger$ & 1.789 & 1.783 & -0.006 \\
\hline 220 & 1.261 & 1.261 & 0.000 \\
\hline 311 & 1.079 & 1.075 & -0.004 \\
\hline $222 f$ & 1.021 & 1.030 & 0.009 \\
\hline 400 & 0.894 & 0.892 & -0.002 \\
\hline 331 & 0.817 & 0.818 & 0.001 \\
\hline $420 \dagger$ & 0.794 & 0.798 & 0.004 \\
\hline 422 & 0.727 & 0.728 & 0.001 \\
\hline 333 & 0.686 & 0.686 & 0.000 \\
\hline 511 & & 0.686 & \\
\hline 440 & 0.630 & 0.631 & 0.001 \\
\hline 531 & 0.597 & 0.603 & 0.006 \\
\hline $600 t$ & & 0.595 & \\
\hline $442 \dagger$ & & 0.595 & \\
\hline 620 & 0.565 & 0.564 & -0.001 \\
\hline 533 & 0.536 & 0.544 & 0.008 \\
\hline $622 \dagger$ & & 0.538 & \\
\hline 444 & & 0.515 & \\
\hline 551 & 0.494 & 0.499 & 0.005 \\
\hline 711 & & 0.499 & \\
\hline $640 \dagger$ & & 0.494 & \\
\hline 642 & 0.472 & 0.477 & 0.005 \\
\hline 553 & 0.460 & 0.464 & 0.004 \\
\hline 731 & & 0.464 & \\
\hline 800 & & 0.446 & \\
\hline 733 & 0.430 & 0.436 & 0.006 \\
\hline $644 t$ & & 0.433 & \\
\hline
\end{tabular}
through-focus series was recorded for several nanocrystals in the irradiated graphite residues. Contrast reversal as expected for diamond was observed. Figure 5 illustrates a plot of the through-focus series performed on the nanocrystal shown in Figure 3. The measured spacing between contrast reversals was found to be $23.3 \pm 3.0 \mathrm{~nm}$, and this was determined from a fast Fourier transform (FFT) analysis of the a-C film. This is in good agreement with $25.7 \mathrm{~nm}$ that was calculated by multi-slice image simulations of diamond. From the electron diffraction and high-resolution imaging data, there can be little doubt that the crystallography of the nanocrystals is that of diamond.

Elemental analysis using an EDXS on the regions containing high nanocrystal concentrations indicated predominantly $\mathrm{Si}, \mathrm{O}$, and $\mathrm{Cu}$ (from the support film and grid) as shown in Figure 6. The only other elements present are $\mathrm{C}, \mathrm{Cl}$, and $\mathrm{S}$. The $\mathrm{Cl}$ and $\mathrm{S}$ are relicts of the acid treatments in the form of amorphous and nanocrystalline precipitates. The $\mathrm{Cl}$-rich nanocrystalline material is 


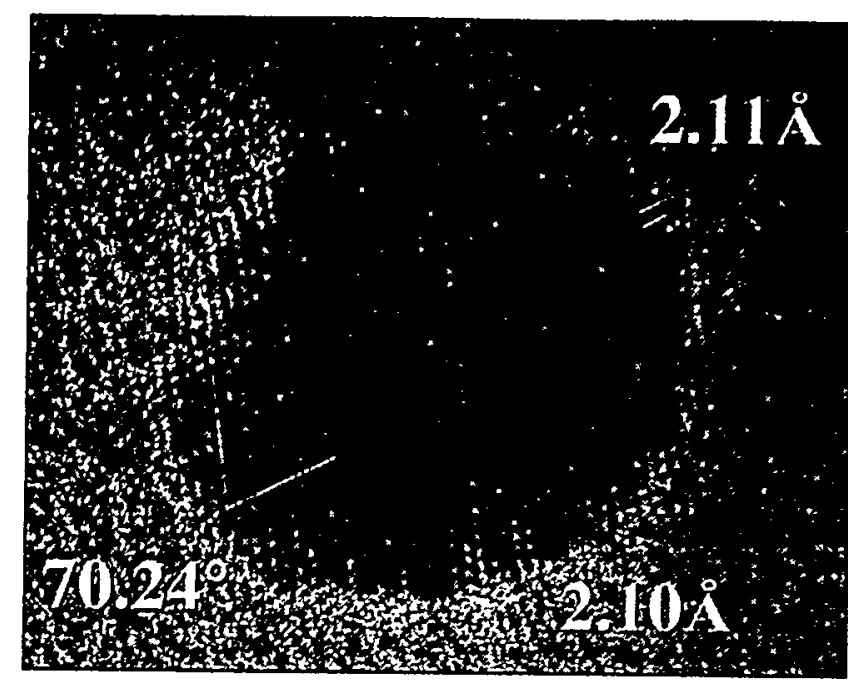

Figure 3: High-resolution lattice image of a twinned nanocrystal in the acid dissolution residue of the ionirradiated graphite. For diamond [011], the (111) plane spacing is $2.06 \AA$ and the angle is $70.5^{\circ}$. extremely beam sensitive and exhibits lattice images distinctly different from diamond.

Our observations indicate the presence of nanodiamonds in the ion irradiated graphite acid residue. This is based on the combination of their electron beam stability, chemical resistance to harsh oxidizing acids, electron diffraction, and lattice images.

The acid dissolution residues of the unirradiated graphite (control) contain several orders of magnitude less nanocrystalline material than the irradiated graphite acid residues. Some of the nanocrystals in the control residue exhibited high-resolution lattice images consistent with that of diamond. However, because of the low concentration of these nanocrystals, it is not possible to identify them as diamond with high confidence.

Therefore, our results indicate that the $400 \mathrm{MeV} \mathrm{Kr}$ ion irradiations of bulk graphite at room temperature do form nanodiamonds. Assuming that recovery by acid dissolution was $75 \%$ effective and $1 \mu \mathrm{g}$ of diamonds with a mean diamond size of $4 \mathrm{~nm}$ were produced, then a rough estimate of diamond yield is $\sim 0.08$ nanodiamonds/ion. In comparison, the diamond yield estimated in the U-rich carbonaceous material was 0.14 nanodiamonds/fission fragment [6]. This supports the speculation that ion irradiation formed the nanodiamonds in the natural material.

\section{SUMMARY}

Acid dissolution residues ( $<3 \mathrm{ppm}$ of bulk)

\section{White Atom-pairs}
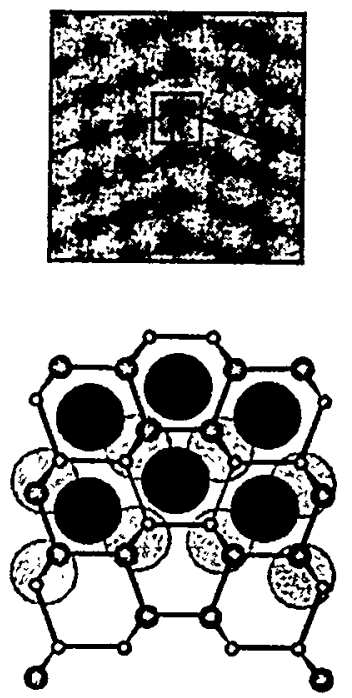

Black Atom-pairs
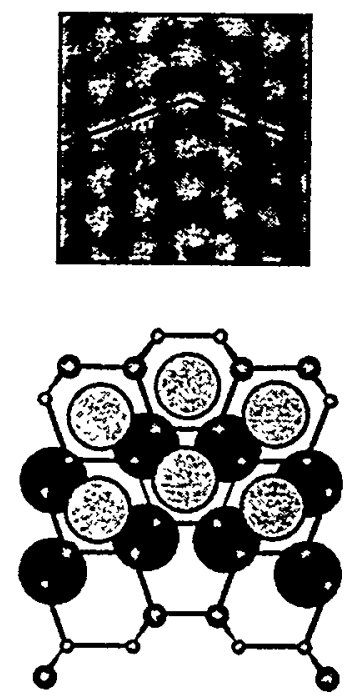

Figure 4: Lattice image of a twin interface in diamond under two defocus conditions which exhibit reversed contrast. Atomic models of the diamond structure are also illustrated.
Through-Focus Series

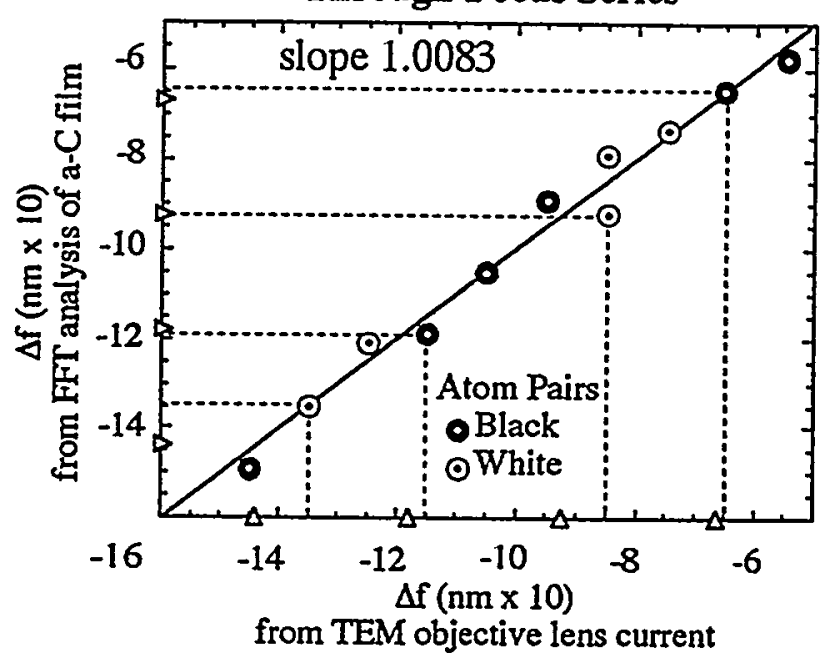

Figure 5: Dependence of image contrast on defocus. The line is a least squares fit and should have slope $=1$ if the microscope is calibrated correctly. The defocus where reversal of image contrast is observed is indicated by the dashed lines, whereas the triangles along the axes denote calculated values. 
Energy Dispersive X-ray Spectroscopy

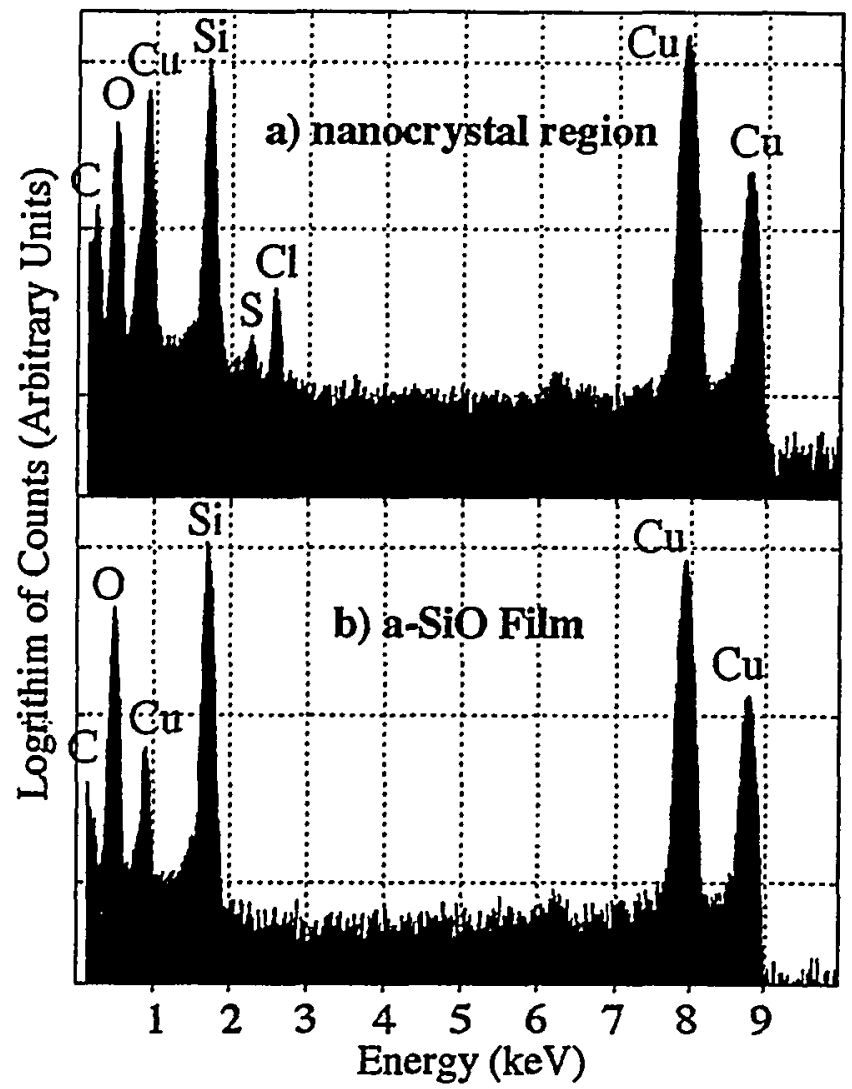

Figure 6: EDXS spectra of a) collections of nanocrystals in the ion-irradiated graphite acid residue and b) a-SiO film containing no nanocrystals. The carbon content in the spectra shown in b) gives an indication of the amount of hydrocarbon build up during measurement. of $400 \mathrm{MeV} \mathrm{Kr}$ ion-irradiated polycrystalline graphite were found to contain nanodiamonds. The identification of nanocrystals in the residues as diamonds is based on their electron beam stability, chemical resistance to harsh oxidizing acids, electron diffraction patterns, and high-resolution lattice images (including lattice spacings and angles as well as throughfocus behavior). Each of these points by themselves cannot provide an unambiguous identification as diamond, but taken together, they do provide strong compelling evidence.

The acid dissolution residues of the unirradiated (control) graphite contain several orders of magnitude less nanocrystalline material. Therefore, our results indicate that the $400 \mathrm{MeV} \mathrm{Kr}$ ion irradiations of bulk graphite at room temperature do form nanodiamonds.

\section{ACKNOWLEDGMENTS}

The ion irradiations were performed in the ANL ATLAS facility and the electron microscopy was performed in the ANL Electron Microscopy Center. The authors thank B. Glagola of ATLAS for assistance in the ion irradiations. The authors also thank

P. Baldo for help in the design of sample stages as well as R. Csencsits and N. Zaluzec for valuable assistance. This research was supported by U.S. DOE under BES contract W-31-109ENG-38 and NASA grant NAG5-4297.

\section{REFERENCES}

1. F. P. Bundy and J. S. Kasper, J. Chem. Phys., 46, 3437-3446 (1967).

2. N. R. Greiner, D. S. Phillips, J. D. Johnson, and F. Volk, Nature, 333, 440-442 (1988).

3. Y. Bar-Yam and T. D. Moustakas, Nature, 342, 786-X X X (1989).

4. F. Banhart and P. M. Ajayan, Nature, 382, 433-435 (1996).

5. P. Wesolowski, Y. Lyutovich, F. Banhart, H. D. Carstanjen, and H. Kronmuller, Appl. Phys. Lett., 71, 1948-1950 (1997).

6. T. L. Daulton and M. Ozima, Science, 271, 1260-1263 (1996).

7. J. P. Biersack, and L. G. Haggmark, J. Nucl. Inst. and Methods, 174, 257-X XX (1980).

8. S. Amari, R. S. Lewis, and E. Anders, Geochim. Cosmochim. Acta, 58, $459-470$ (1994).

9. R. S. Lewis, E. Anders, and B. T. Draine, Nature, 339, 117-121 (1989).

10. M. Frenklach, R. Kematick, D. Huang, W. Howard, K. E. Spear, A. W. Phelps, and R. Koba, J. Appl. Phys., 6 6, 395 (1989).

11. P. R. Buerki and S. Leutwyler, J. Appl. Phys., 69, 3739-3744 (1991).

12. K. S. Harshavardhan, R. S. Yalamanchi, and L. K. Rao, Appl. Phys. Lett., 55, 351-XXX (1989).

13. T. L. Daulton, D. D. Eisenhour, T. J. Bernatowicz, R. S. Lewis, and P. R. Buseck, Geochim. Cosmochim. Acta, 6 0, 4853-4872 (1996). 